Supplement of

\title{
Adaptation and performance assessment of a quantum and interband cas- cade laser spectrometer for simultaneous airborne in situ observation of $\mathrm{CH}_{4}, \mathrm{C}_{2} \mathrm{H}_{6}, \mathrm{CO}_{2}, \mathrm{CO}$ and $\mathrm{N}_{2} \mathrm{O}$
}

Julian Kostinek et al.

Correspondence to: Julian Kostinek (julian.kostinek@dlr.de)

The copyright of individual parts of the supplement might differ from the CC BY 4.0 License. 


\section{S1 Compatibility \& Comparison with other instruments}

We have computed the differences between flasks and the CRDS in the same way they have been computed for the QCLS in the main manuscript by interpolating high frequent in situ data to the flask end fill times. This has been done to get an idea on what spread can be expected due to the long flask sampling time compared to the fast measurement cycles of CRDS and

5 QCLS. Although the spread in Fig. S1 might be slightly bigger for the QCLS compared to the CRDS it remains unclear if this is due to higher sampling rate and/or response time of the QCLS.
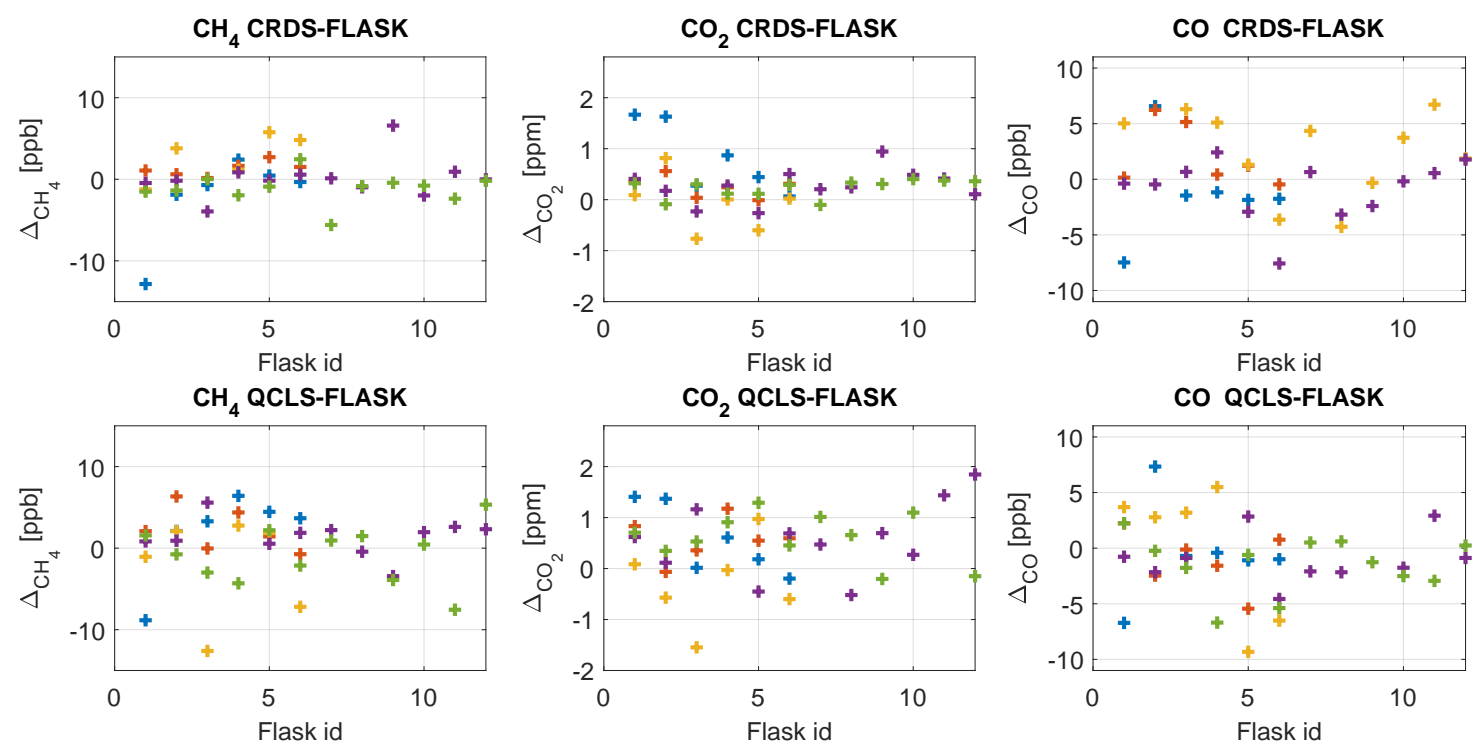

Figure S1. Comparison of CRDS (upper row) and QCLS (lower row) derived mole fractions to flask samples. Interpretation of the errors against flask samples is difficult for high-variability flight segments, due to the large flask sampling time. The residual plots show color-coded data from 5 typical flights on 10/03/2017, 10/11/2017, 10/14/2017, 10/18/2017 and 10/20/2017.

\section{S2 Cross-sensitivities}

There was doubt if the water vapor correction is deteriorating compatibility between instruments. Figure S2 shows cross sensitivities of the QCLS-CRDS residuals for the flight highlighted in the original manuscript (10/03/2017) with respect to cell pressure, cell temperature and water vapor after correcting for a constant bias. The residuals have been computed by linearly interpolating the higher frequency QCLS to the CRDS time scale, due to different sampling times and patterns. From this figure, we would argue, that the water vapor correction (center row in Figure S2) is not systematically deteriorating compatibility between the instruments.

\section{S3 Large bias in $\mathrm{CO}_{2}$}

15 Explaining the large bias in retrieved $\mathrm{CO}_{2}$ requires an estimation of the influence of the isotopic composition of the working standards and the sampled air within this study. We have used working standards of synthetic nature from Air Liquide due to the large amount of needed calibration gas. Usually these are produced with $\mathrm{CO}_{2}$ from natural gas \& oil combustion processes. We determined the $\mathrm{CH}_{4}$ and $\mathrm{CO}_{2}$ values of each working standard gas cylinder using a Picarro G-1301m. This has the drawback 

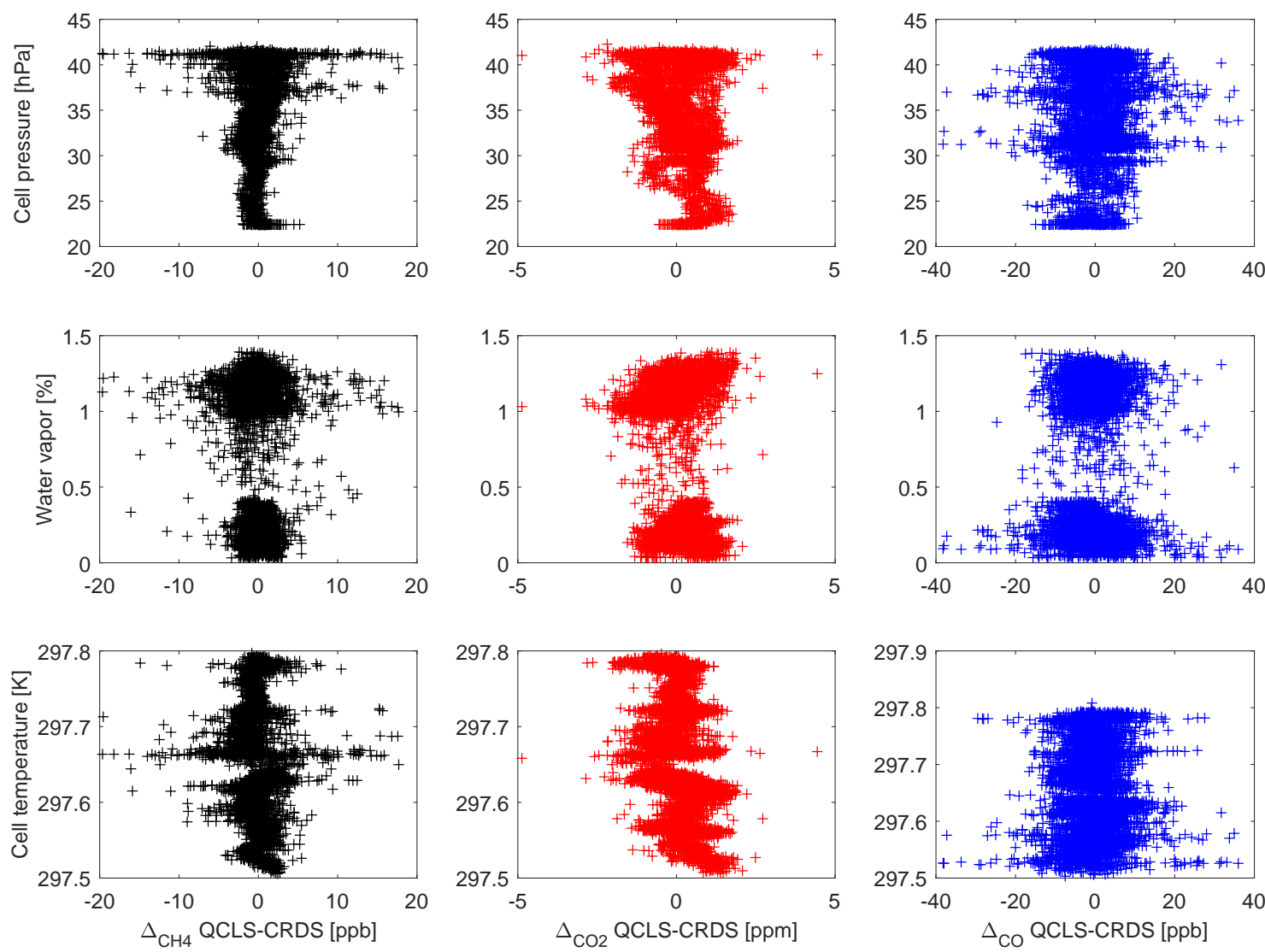

Figure S2. Cross sensitivities of the QCLS-CRDS residuals for the flight highlighted in the original manuscript (10/03/2017) with respect to cell pressure, cell temperature and water vapor after correcting for a constant bias. Due to different sampling times and patterns the higher frequency QCLS data have been linearly interpolated to the CRDS time scale.

that we do not know the isotopic composition of our working standards. The reason why we did not send our working standards to a central lab is because the influence of the isotopic composition had been considered negligible at this stage (Chen et al., 2010). It was only in late summer 2018, when we found that the instrument was using a ${ }^{13} \mathrm{C}^{16} \mathrm{O}_{2}$ line to derive ambient $\mathrm{CO}_{2}$. We assume the large bias originating from differences in isotopic composition in our working standards relative to the natural terrestrial abundances. This requires an estimate on the possible influence which will be given here.

It is commonly assumed that differences in isotopic composition only make up for errors on the order of $0.1 \mathrm{ppm}$ in $\mathrm{CO}_{2}$. This is true if measuring the primary isotopologue, as done with the Picarro CRDS. If the $\mathrm{CO}_{2}$ concentration is derived from the secondary isotopologue $\left({ }^{13} \mathrm{C}^{16} \mathrm{O}_{2}\right)$ the influence of isotopic composition is much larger. Let us take the $\delta^{13} C=-8.6$ $\%$ reported for the NOAA standard Cert.-Nr. CB11361 as an example to estimate the influence of isotopic composition on retrieved mole fractions. Per definition $\delta^{13} C$ is given by

$\delta^{13} C=\left(\frac{R_{x}}{R_{v p d b}}-1\right) \times 1000$ 
Inserting the values from above and re-arranging this equation yields

$R_{\text {noaa }}=\left(1-8.6 \times 10^{-3}\right) \times R_{v p d b}$

Inserting the standard ratio for the reference materials of the Vienna Pee Dee Belemnite $R_{v p d b}=0.011180 \pm 0.000028$ (Tohjima et al., 2009; Chen et al., 2010) yields the corresponding isotopic ratio of

$5 \quad R_{\text {noaa }}=\left(1-8.6 \times 10^{-3}\right) \times 0.011180=0.011083$

According to HITRAN the primary isotopologue and secondary isotopologue together make up $99.5261 \%$ of atmospheric $\mathrm{CO}_{2}$. To satisfy both equations we obtain an abundance of 0.984350 primary isotopologue and $0.010910{ }^{13} \mathrm{C}^{16} \mathrm{O}_{2}$ for the NOAA standard dealt with here. However, spectral line intensities $S_{i j}$ as defined on https://hitran.org/docs/definitions-and-units/are weighted according to the natural terrestrial abundances reported in HITRAN. Retrieved mole fractions are thus scaled by their terrestrial natural abundance from HITRAN. We'll first compute the unscaled ${ }^{13} \mathrm{C}^{16} \mathrm{O}_{2}$ using the natural abundance from HITRAN (98.4204\% primary and $1.1057 \%$ secondary $\mathrm{CO}_{2}$ isotopologue) from a hypothetical $400 \mathrm{ppm}$ background (with a natural isotopic composition as defined in HITRAN) resulting in

${ }^{13} \mathrm{CO}_{2, \text { unscaled }}=400 * 0.011057=4.4228$

We can now estimate the influence of the different isotopic composition (NOAA example) from above by scaling the unscaled ${ }^{13} \mathrm{CO}_{2}$,unscaled with the secondary isotopologue abundance computed above. For the given absopriotn line, a retrieval algorithm based on HITRAN will retrieve an abundance of $\mathrm{CO}_{2, \text { retrieved }}$ according to

$C O_{2, \text { retrieved }}=\frac{4.4228}{0.010910}=405.4 \mathrm{ppm}$

From this example we see that the small perturbation in isotopic composition already has an impact of $\mathbf{5 . 4} \mathbf{~ p p m}$ in retrieved $\mathrm{CO}_{2}$.

20 Chen et al., 2010 reported on synthetic air with added $\mathrm{CO}_{2}$ from burned petroleum or natural gas with $\delta^{13} C=-37 \pm 11$ $\%$. Using this value, and repeating the math from above, we obtain a change of $\mathbf{1 7 . 2} \mathbf{~ p p m}$ in retrieved mole fractions resulting solely from a different isotopic composition. Given this estimate, we find that, precise knowledge of the $\delta^{13} \mathrm{C}$ of the working standards and the sampled air is needed to enhance $\mathrm{CO}_{2}$ compatibility when operating on the $2227.604 \mathrm{~cm}^{-1}$ ${ }^{13} \mathrm{C}^{16} \mathrm{O}_{2}$ absorption line. In the abscence of other error sources, achieving WMO compatibility using this absorption line at 25 ambient $\mathrm{CO}_{2}$ concentrations of $400 \mathrm{ppm}$ requires the sum of $\delta^{13} \mathrm{C}$ from the working standards and the sampled air to be known better than $4.9 \%$ o. In reality other error sources are not negligible, which will further reduce the stated margin. 


\section{S4 Calibration cycles}

We included distribution diagrams of the calibration cycles during the flight highlighted in the main manuscript (10/03/2017) in Figure S3.
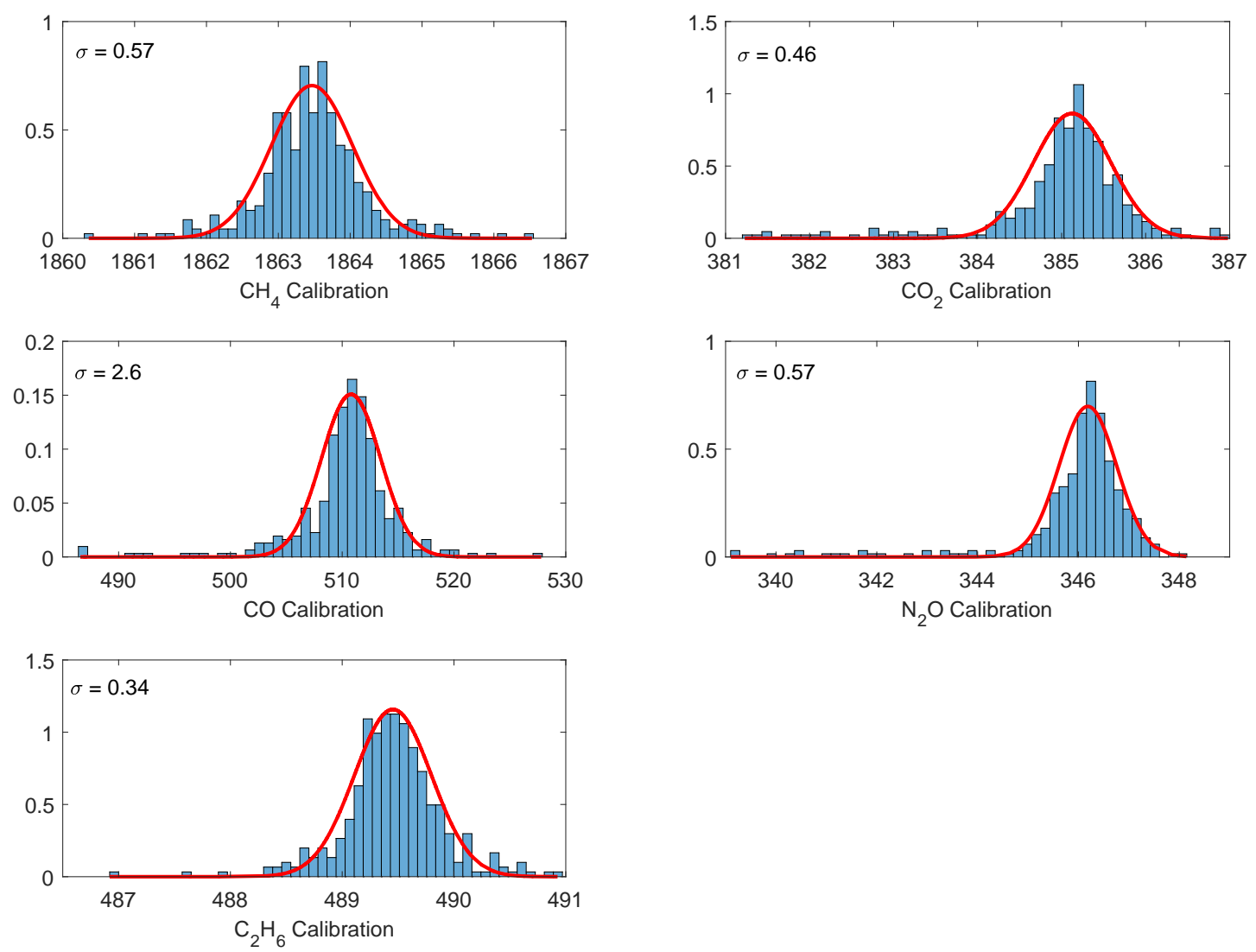

Figure S3. Distribution diagrams of the calibration gas measurements during the flight highlighted in the main manuscript on Oct. 3, 2017. 